\title{
DANOS DE Pygiopachymerus lineola (Chevrolat, 1871) (COLEOPTERA: CHRYSOMELIDAE: BRUCHINAE) EM SEMENTES DE CHUVA-DE-OURO (Cassia fistula L.) UTILIZADAS NA ARBORIZAÇÃO URBANA DE SANTA MARIA, RS
}

\author{
Jardel Boscardin $^{12}$; Ervandil Corrêa Costa ${ }^{3}$; Juliana Garlet ${ }^{4}$; Augusto Bolson Murari ${ }^{5}$
}

\section{RESUMO}

O presente trabalho objetivou identificar e caracterizar os danos ocasionados a sementes de Cassia fistula L. (Fabaceae) (chuva-de-ouro) por um inseto granívoro. Com este intuito, de setembro a outubro de 2010 foram coletadas 100 vagens, diretamente de 10 árvores utilizadas na arborização urbana de Santa Maria, Rio Grande do Sul, Brasil. Posteriormente, as vagens foram encaminhadas ao laboratório e depositadas em cubas de vidro cobertas por uma tela. Após o surgimento dos adultos, foi quantificado o total de sementes sadias, chochas e predadas contido nas vagens. O conteúdo das sementes, que foi consumido pelo inseto, foi obtido pela diferença de peso entre sementes sadias predadas, pesadas em cinco lotes contendo 100 sementes cada. As médias dos lotes de sementes sadias e predadas foram comparadas através do teste de Tukey, em nível de $5 \%$ de probabilidade. A partir dos dados levantados, constatou-se que Pygiopachymerus lineola (Chevrolat, 1871) (Coleoptera: Chrysomelidae: Bruchinae) foi responsável pelo ataque de 53,7 \% de um total de 3.991 sementes avaliadas, com um consumo médio significativo de $64,6 \%$ do peso destas sementes. Desta forma, o referido ataque compromete a germinação das sementes de forma irreversível, afetando a produção de mudas.

Palavras-chave: entomologia florestal; árvore ornamental; inseto sitófago

\section{DAMAGES OF Pygiopachymerus lineola (Chevrolat, 1871) (COLEOPTERA: BRUCHIDAE) IN CHUVA- DE-OURO (Cassia fistula L.) SEEDS USED IN URBAN ARBORIZATION OF SANTA MARIA, RS}

\section{ABSTRACT}

This study aimed to identify and characterize the damage caused to seeds of Cassia fistula L. (Fabaceae) (rainof-gold) by a granivorous insect. For this purpose, from September to October of 2010, 100 pods were collected directly from 10 trees used in the urban arborization of Santa Maria, Rio Grande do Sul, Brazil. Later, the pods were sent to the laboratory and placed in glass vats covered with a screen. After the appearance of the adults, the total of healthy, predated and empty seeds contained in the pods was quantified. The content of seeds, which was consumed by the insect, was obtained by weight difference between healthy and predated seeds, weighed into five lots containing, each one, 100 seeds. The average of the lots of healthy and predated seeds was compared using the Tukey test at 5\% level of probability. From the data collected, it was found that Pygiopachymerus lineola (Chevrolat, 1871) (Coleoptera: Chrysomelidae: Bruchinae) was responsible for the attack of $53.7 \%$ from a total of 3,991 seeds evaluated, with a significant average consumption of $64.6 \%$ by weight of these seeds. Thus, this attack compromises the germination of the seeds irreversibly, affecting the production of seedlings.

Keywords: forest entomology; ornamental tree; granivorous insect

\footnotetext{
${ }^{1}$ Eng. Florestal, Mestrando em Eng. Florestal pelo Programa de PG em Eng. Florestal da Univ. Fed. Santa Maria, Depto. de Defesa Fitossanitária, Santa Maria, RS boscardinj@gmail.com

${ }^{2}$ Data de Recebimento - 29/11/2011 - Data de publicação: 15/12/2012

${ }^{3}$ Eng. Agrônomo, Prof. Titular da Univ. Fed. Santa Maria, Depto. de Defesa Fitossanitária, Santa Maria, RS ervandilc@gmail.com

${ }^{4}$ Eng. Florestal, Doutoranda em Eng. Florestal pelo Programa de PG em Eng. Florestal. Univ. Fed. Santa Maria, Depto. de Defesa Fitossanitária, Santa Maria, RS julianagarlet@yahoo.com.br

${ }^{5}$ (In memoriam) Eng. Florestal, Mestre em Eng. Florestal, Univ. Fed. Santa Maria, Depto. de Defesa Fitossanitária, Santa Maria, RS.
} 


\section{INTRODUÇÃO}

A Cassia fistula L., denominada vulgarmente de cassia-imperial, cássia-fistula, canafístula, chuvade-ouro, e acácia imperial, pertence à família Fabaceae, e é originária da Índia. É uma árvore de pequeno porte, que pode atingir de 10 a 15 metros de altura, seu florescimento, de coloração amarelaouro ou amarela-limão, ocorre entre os meses de setembro e outubro, produz vagens lenhosas, cilíndricas, indeiscentes, marrom-escuras ou pretas, quebradiças. As sementes são de coloração castanha, envolvidas por uma mucilagem preta, luzidias, com aroma de alcaçuz, de uso medicinal e aromatizante (SANTOS; TEIXEIRA, 2001; LORENZI et al., 2003).

Por apresentar uma floração exuberante, a Cassia fistula L. constitui-se em uma espécie arbórea ornamental bastante utilizada na arborização urbana, e devida sua estrutura e porte é indicada apenas para canteiros centrais. Não aceita poda, e é facilmente atacada por lagartas (SANTOS; TEIXEIRA, 2001). A obtenção de mudas ocorre principalmente através do plantio de sementes, e também por meio de propagação vegetativa com estacas (BACKES; IRGANG, 2002).

Para a obtenção de mudas de qualidade e de baixo custo, é necessário que se tenha além de sementes sadias, porcentagem de germinação satisfatória. Espécies como a Cassia fistula L. possuem o tegumento da semente espesso e/ou impermeável que impede a absorção de água (PEREZ, 2004). Sendo necessária, portanto, a quebra de dormência, como recomendam Lopes et al. (2003), através da escarificação mecânica e a imersão em ácido sulfúrico por no máximo 60 minutos.

Em espécies ornamentais encontram-se associados insetos, parasitóides, predadores, bem como patógenos. Um dos componentes desse nicho é a família Bruchidae, que se constitui de insetos associados a sementes de várias espécies vegetais de importância agrícola, podendo afetar a germinação e a qualidade das sementes, principalmente quando armazenadas (CARVALHO; FIGUEIRA, 1999).

Além de importantes para a agricultura, esses insetos sitófagos também causam danos em frutos e sementes de espécies florestais, tanto nativas quanto exóticas, incluindo as utilizadas como ornamentais. Nascimento (2009), por exemplo, verificou o ataque em sementes de plantas de Albizzia lebbeck (Benth.) (albízia), utilizadas na arborização de ruas e avenidas de cidades do Estado do Rio de Janeiro, ocasionado pelas espécies Bruchidius sp., Merobruchus paquetae Kingsolver, 1980 (Coleoptera: Bruchidae) e Stator limbatus (Horn, 1873) (Coleoptera: Chrysomelidae: Bruchinae). Na maioria das vezes, práticas inadequadas na poda de espécies arbóreas em vias públicas fazem com que estas sofram estresse e tornem-se mais susceptíveis a ocorrência de pragas e doenças.

Diante do exposto, o presente estudo objetivou identificar e quantificar os danos da espécie responsável pelo ataque a sementes de Cassia fistula L.. 


\section{MATERIAIS E MÉTODOS}

\section{Localização e caracterização da área de estudo}

O estudo foi realizado em árvores de Cassia fistula L., de diferentes idades, pertencentes à arborização urbana do município de Santa Maria, localizado na região fisiográfica da Depressão Central do Rio Grande do Sul, coordenadas UTM 0230270 S e 6711452 W, Datum WGS84.

O clima da região, segundo a classificação de Köppen, é do tipo Cfa, subtropical úmido, sem estiagens. Tem como características climáticas principais a temperatura média anual de $19^{\circ} \mathrm{C}$, precipitação média anual de 1.769 mm e umidade relativa do ar de 82 \% (MORENO, 1961).

\section{Coleta e triagem do material}

Nos meses de setembro e outubro de 2010, foram coletadas 100 vagens diretamente de dez árvores de Cassia fistula L., de acordo com a disponibilidade de frutos e viabilidade de coleta, localizadas na Avenida Medianeira, Avenida Fernando Ferrari e Parque Itaimbé, na cidade de Santa Maria, Rio Grande do Sul.

Depois de realizadas as coletas, os frutos devidamente embalados foram encaminhados até o Laboratório de Entomologia do Departamento de Defesa Fitossanitária da Universidade Federal de Santa Maria (UFSM), onde se procedeu a avaliação do dano. Para tanto, as vagens foram acondicionadas em cubas de vidro, recobertas por uma tela na abertura superior, de forma que, os adultos ao emergirem ficassem retidos. As cubas permaneceram no laboratório, em local arejado, e em condição ambiente de temperatura e umidade relativa.

Após a emergência dos adultos, com o auxilio de uma pinça, as sementes foram removidas das vagens e classificadas em sadias (com aspecto normal e livre de ataque de insetos), sementes chochas (com aspecto de formação anormal) e atacadas por insetos (SARI; RIBEIRO-COSTA, 2005). Exemplares dos adultos foram enviados para a Dra. Cibele Stramare Ribeiro-Costa, do Departamento de Zoologia da Universidade Federal do Paraná, Brasil, para identificação.

\section{Análise dos dados}

A partir do total de sementes encontradas nas vagens $(\mathrm{N})$, foi verificada a percentagem (\%) de sementes sadias (Ss), chochas (Sc) e sementes atacadas (Sa) pelo inseto sitófago. Para o cálculo da porcentagem utilizou-se a seguinte equação:

$$
\%=\frac{(S s, S c, S a \times 100)}{N}
$$

O conteúdo (massa) da semente consumida pelo inseto foi obtido pela diferença de peso (em gramas) entre sementes sadias e sementes predadas. Para tanto, as sementes sadias e predadas foram separadas em cinco lotes contendo 100 unidades cada, e pesadas em balança eletrônica de precisão, marca MarK, modelo 3500.

Os lotes de sementes, sadias e predadas, procedentes do mesmo período de coleta, foram analisados, e os resultados obtidos submetidos à análise estatística pelo teste de Tukey ao nível de $5 \%$ de probabilidade, com o auxilio do programa 


\section{RESULTADOS E DISCUSSÃO}

A espécie encontrada predando sementes de chuvade-ouro, foi identificada como Pygiopachymerus lineola (Chevrolat, 1871) pertencente a ordem
Coleoptera, família Chrysomelidae, subfamília Bruchinae (Figura 1).

Figura 1. Adulto de Pygiopachymerus lineola (Chevrolat, 1871) (Coleoptera: Chrysomelidae: Bruchinae), encontrado atacando sementes de Cassia fistula L (chuva-de-ouro). em Santa Maria, RS. Setembro e outubro de 2010

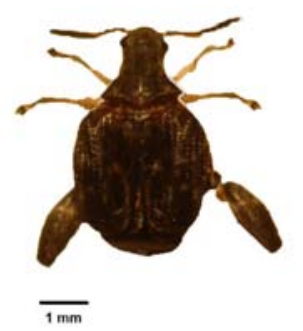

Pygiopachymerus lineola (Chevrolat, 1871) (Coleoptera: Chrysomelidae: Bruchinae), possui a característica de se desenvolver no interior das sementes, e emergir após atingir o estágio adulto. Em um estudo realizado por Ribeiro-Costa e Costa (2002), sobre a postura de Pygiopachymerus lineola em Cassia leptophylla, as autoras verificaram que os ovos são depositados pela fêmea sobre as vagens e a postura consiste de três ou mais ovos, distribuídos de forma aleatória na superfície da vagem, sendo observadas em alguns casos, posturas em áreas com o tegumento ligeiramente danificado. Seu ciclo de vida, em condições de laboratório predando Cassia javanica L., segundo Carvalho e Figueira (1999) é de 58,4 \pm 9,18 dias.

Este bruquídeo já foi registrado predando sementes de diversas espécies arbóreas, também pertencentes ao gênero Cassia e utilizadas como ornamentais. No Rio de Janeiro, foi encontrado predando sementes da própria Cassia fistula L., Cassia grandis L. e Cassia javanica L. (CARVALHO; FACRE, 1987). Casari e Teixeira (1997), verificaram a presença do bruquídeo em sementes de outras espécies, além das Cassia fistula e Cassia javanica, também pertencentes à subfamília Caesalpiniaceae, como Cassia ferruginea, Cassia leptophylla, Peltophorum dubium e Senna multijuga. Provavelmente este seja o primeiro registro da incidência deste bruquídeo em Cassia fistula para o Rio Grande do Sul.

Quanto aos danos ocasionados pelo bruquídeo, das 100 vagens avaliadas no presente trabalho, foram encontradas 3.991 sementes, destas, 849 sadias, 1.000 chochas e 2.142 atacadas por Pygiopachymerus lineola, correspondendo a 21,3 $\%$, 25,0 \% e 53,7 \%, respectivamente. A perda decorrente do ataque apresentou-se mais severa que as verificadas por Ferraz e Carvalho (2001), que em 
seu trabalho, realizado também com Pygiopachymerus lineola em sementes de Cassia fistula, encontraram 62,8\% de sementes sadias, 32,2 $\%$ de sementes atacadas e 5,0\% de sementes chochas. O mesmo ocorreu para Senna multijuga, onde Sari e Ribeiro-Costa (2005) observaram uma perda de somente $12 \%$ das sementes analisadas, sendo que os restantes $62,0 \%$ foram representados por sementes sadias e $26,0 \%$ de sementes chochas.

As larvas consumiram os cotilédones e a testa das sementes da chuva-de-ouro (Figura 2), comprometendo sua germinação de forma irreversível. Verificou-se este mesmo dano em sementes de acácia-negra atacados por Stator limbatus (OLIVEIRA; COSTA, 2009).

Figura 2. Semente de Cassia fistula L. (chuva-de-ouro) sadia (A) e semente atacada (B) por Pygiopachymerus lineola (Chevrolat, 1871) (Coleoptera: Chrysomelidae: Bruchinae) em Santa Maria, RS. Setembro e outubro de 2010

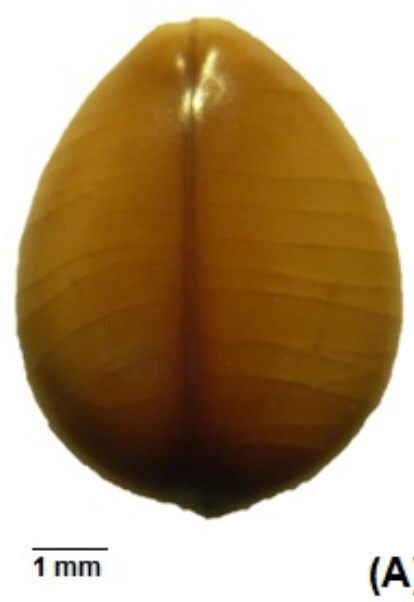

(A)

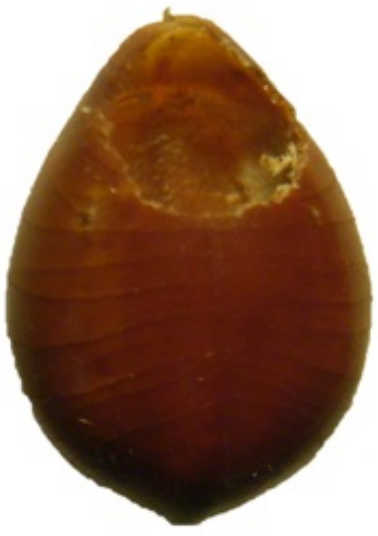

$\overline{1 \mathrm{~mm}}$

(B)
Foi verificada diferença significativa entre o peso de sementes sadias e predadas, pelo teste de Tukey a 5 \% de probabilidade de significância.

O peso médio dos lotes de sementes de chuva-deouro, sadias e predadas pelo bruquídeo foi de 10,384 g e 3,674 g, respectivamente. Em média 6,710 g de substrato foi consumido de cada lote contendo 100 sementes, atingindo assim, um percentual de $64,6 \%$ do peso das sementes de chuva-de-ouro. Esses dados corroboram com Oliveira e Costa (2009) que encontraram um percentual de $60,4 \%$ no consumo de sementes de Acacia mearnsii por Stator limbatus. Em contrapartida, Donato et al. (2010), verificaram um consumo de 28,96 \% das sementes de Enterolobium contortisiliquum atacadas por Caryedes sp. (Coleoptera: Bruchidae).

A diferença estatística encontrada demonstra a severidade do dano causado por esse inseto, que compromete a germinação das sementes de chuvade-ouro, inviabilizando sua utilização para produção de mudas. 


\section{CONCLUSÃO}

Os danos causados pelo ataque do bruquídeo identificado como Pygiopachymerus lineola, em sementes de Cássia fistula L., afetam de forma negativa sua germinação, comprometendo a produção de mudas via sexuada desta espécie.

\section{AGRADECIMENTOS}

Agradecemos a Doutora Cibele Stramare Ribeiro-

Costa, do Departamento de Zoologia da
Universidade Federal do Paraná pela identificação da espécie.

\section{REFERÊNCIAS BIBLIOGRÁFICAS}

BACKES, P.; IRGANG, B. Árvores do Sul. Guia de identificação \& interesse Ecológico. As principais espécies nativas Sul-Brasileiras. Santa Cruz do Sul: Instituto Souza Cruz, 2002.

CARVALHO, A. G.; FIGUEIRA, L. K. Biologia de Pygiopachimerus lineola (Chevrolat, 1871) (Coleoptera: Bruchidae) em frutos de Cassia javanica L. (Leguminosae: Caesalpinioideae). Floresta e Ambiente, v. 6, n. 1, p.83-87, 1999.

CARVALHO, A. G.; FACRE, J. R. N. Aspectos biológicos e danos de Pygiopachimerus lineola (Chevrolat, 1871) (Coleoptera: Bruchidae) em frutos de Cassia spp. In: CONGRESSO BRASILEIRO DE ENTOMOLOGIA. Resumos... Campinas, SP. Sociedade Entomológica do Brasil, v.1, n.32, 1987.

CASARI, S. A.; TEIXEIRA, E. P. Descriptions and biological notes of final larval instar and pupa of some seed beebtles (Coleoptera: Bruchidae). Annales de la Société Entomologique de France, v. 33, n. 3, p. 295-321, 1997.

DONATO, D. B.; FONSECA, A. G.; ASSIS JÚNIOR, S. L.; MACHADO, E. L. M. BISPO, D. F. A. Dano de Caryedes sp. (COLEOPTERA: BRUCHIDAE) e seus Reflexos na Propagação de Enterolobium contortisiliquum (LEGUMINOSAE). Floresta e Ambiente, v. 17, n. 2, p. 118-123, 2010. 
FERRAZ, F. C.; CARVALHO, A. G. Ocorrência e danos por Pygiopachymerus lineola (Chevrolat, 1871) (Coleoptera: Bruchidae) em frutos de Cassia fistula L. no campus da Universidade Federal Rural do Rio de Janeiro. Biotemas, v. 14, n. 1, p. 137-140, 2001.

LOPES, J. C.; SILVA, G. F.; POSSE, S. C. P.; RUY, J. Geminação e dormência de sementes de Cassia fistula L. Brasil Florestal, n. 78, p. 67-74, 2003.

LORENZI, H.; SOUZA, H. M.; TORRES, M. A. V.; BACHER, L. B. Árvores exóticas no Brasil: madeireiras, ornamentais e aromáticas. São Paulo: Nova Odessa: Instituto Plantarum, 2003.

MORENO, J. A. Clima do Rio Grande do Sul. Porto Alegre: Secretaria da Agricultura, 1961.

NASCIMENTO, L. S. Ecologia de Bruchidae na predação pré-dispersão de sementes de Albizzia lebbeck (Benth.) em arborização. Seropédica, Rio de Janeiro, 2009. 73 f. Dissertação (Mestrado em Ciências) Universidade Federal Rural do Rio de Janeiro, Seropédica, Rio de Janeiro, 2009.

OLIVEIRA, L. S.; COSTA, E. C. Predação de sementes de Acacia mearnsii De Wild. (Fabaceae, Mimosoideae). Biotemas, v. 22, n. 2, p. 39-44, 2009.

RIBEIRO-COSTA, C. S.; COSTA, A. S. Comportamento de oviposição de Bruchidae (Coleoptera) predadores de sementes de Cassia leptophylla Vogel (Caesalpinaceae), morfologia dos ovos e descrição de uma nova espécie. Revista Brasileira de Zoologia, v. 19, (Supl. 1), p. 305-316, 2002.

PEREZ, S. C. J. DE A. Envoltórios. In: FERREIRA, A. G.; BORGHETTI, F (Org.). Germinação: do básico ao aplicado. Porto Alegre: Armed, 2004, p. 125-134.

SANTOS, N. R. Z.; TEIXEIRA, I. F. Arborização de Vias Públicas: Ambiente x Vegetação. Santa Cruz do Sul: Instituto Souza Cruz, 2001.

SARI, L. T.; RIBEIRO-COSTA, C. S; ROPER, J. J. Predação de sementes de Senna multijuga (Rich.) H.S. Irwin \& Barneby (Caesalpinaceae) por bruquíneos (Coleoptera, Chrysomelidae). Neotropical Entomology, v. 34, n. 3, p. 521-525, 2005.

SILVA, F. DE A. S.; AZEVEDO, C. A. V. DE. Principal components analysis in the software Assistat-statistical attendance. In: WORLD CONGRESS ON COMPUTERS IN AGRICULTUMIDADE RELATIVAE, 7., 2009, Reno, USA: Anais... Reno, USA: American Society of Agricultural and Biological Engineers, 2009.

DANOS DE Pygiopachymerus lineola (Chevrolat, 1871)... 